\title{
Avaliação da resposta aos esquemas de tratamento reduzidos para malária vivax
}

\author{
Assessment of the response to reduced treatment schemes \\ for vivax malaria
}

Nagib Ponteira Abdon', Ana Yecê das Neves Pinto², Rita do Socorro Uchôa das Silva² e José Maria de Souza ${ }^{1,2}$

\begin{abstract}
Resumo Recaídas que podem ocorrer com o tratamento convencional de longa duração para malária por P. vivax, são em parte devido a parcial adesão do paciente ao tratamento. A utilização de esquemas terapêuticos de curta duração pode proporcionar melhor adesão ao tratamento, mantendo a eficácia e tolerância $e$ minimizando efeitos adversos. Com o objetivo de testar 2 esquemas terapêuticos com doses reduzidas de cloroquina para malária por P.vivax, comparando-o ao esquema clássico, os autores estudaram 120 pacientes, diagnosticados pela gota espessa, com idade superior a 12 anos, submetidos a três esquemas de tratamento: esquema l: fosfato de cloroquina (150mg), $25 \mathrm{mg} / \mathrm{kg}$ (dose total), durante três dias (10 $\mathrm{mg} / \mathrm{kg}$ de peso no primeiro dia; $7,5 \mathrm{mg} / \mathrm{kg}$ no segundo e terceiro dias), associada a primaquina $(15 \mathrm{mg}), 0,25 \mathrm{mg} / \mathrm{kg} / \mathrm{dia}$, por quatorze dias; esquema Il: cloroquina, $10 \mathrm{mg} / \mathrm{kg}$, em dose única, associada a primaquina, $0,5 \mathrm{mg} / \mathrm{kg} / \mathrm{dia}$, por sete dias; esquema III: cloroquina, $10 \mathrm{mg} / \mathrm{kg}$ em dose única, associada a primaquina, $0,5 \mathrm{mg} / \mathrm{kg} / \mathrm{dia}$, por cinco dias. A resposta de cura clínica aos três esquemas foi satisfatória. O desaparecimento da tríade sintomática ocorreu no máximo em até 96 horas, e a negativação da parasitemia assexuada ocorreu em até 72 horas, para os três esquemas.

Palavras-chaves: Malária vivax. Tratamento reduzido. Cloroquina.
\end{abstract}

Abstract Relapses may occur with long standard treatment of vivax malaria, and these are caused by incomplete patient's compliance. The use of reduced schedules may further better patient compliance, while maintaining the same efficacy, tolerance and minimal adverse reactions. The objective of this study was to test two schedules with reduced doses of chloroquine for vivax malaria and comparing these with the classical schedule. The authors studied 120 outpatients, with vivax malaria, aged over 12 years, submitted to three therapeutic schemes: scheme l: chloroquine phosphate $(150 \mathrm{mg})$ in a dose of $25 \mathrm{mg} / \mathrm{kg} /$ day for three days $(10 \mathrm{mg} / \mathrm{kg} /$ day in the first day, $7.5 \mathrm{mg} / \mathrm{kg} /$ day in the second and third day), plus primaquine $(15 \mathrm{mg}$ ) in a dose of $0.25 \mathrm{mg} / \mathrm{kg} /$ day for fourteen days; scheme II: chloroquine, in a single dose of $10 \mathrm{mg} / \mathrm{kg}$, plus primaquine in a dose of $0.5 \mathrm{mg} / \mathrm{kg} /$ day for seven days; scheme III: chloroquine, $10 \mathrm{mg} / \mathrm{kg}$ in a single dose plus primaquine in a dose $0.5 \mathrm{mg} / \mathrm{kg} / \mathrm{day}$ for five days. The clinical response to all three therapeutic schemes was satisfactory. The disappearance of malarial symptoms occurred after a maximum 96 hours of treatment, while the assexual parasitaemia clearance occurred within 72 hours, in all therapeutic schemes.

Key-words: Vivax malaria. Reduced treatment. Chloroquine.

A malária produzida pelo Plasmodium vivax, por ser considerada benigna e autolimitada, pode levar à suspeição diagnóstica imprecisa e conseqüentemente a terapêutica imprópria, além de complicações que poderão surgir, sobretudo àquelas relacionadas a anemia. O esquema terapêutico adotado pela Fundação Nacional de Saúde, para o tratamento radical da malária por $P$. vivax, era realizado em quatorze dias. Apesar de eficaz, este esquema prolongado pode ser o responsável pelo excessivo número de casos de abandono do tratamento e conseqüentemente pelo aparecimento das recaídas. Este tratamento convencional da malária por $P$. vivax serviu de modelo para as observações preliminares dos autores, principalmente considerando a observação de ordem prática, de quê, após a primeira dose da cloroquina, os pacientes apresentavam uma redução acentuada, ou até mesmo o desaparecimento da parasitemia assexuada. Por outro lado, os pacientes que abandonavam o tratamento, portanto não utilizando a primaquina durante quatorze dias e retornavam ao serviço, apresentavam exame de pesquisa de plasmódio negativo. Nesses casos o acompanhamento realizado até o final dos cento e oitenta dias, não demonstrava parasitemia positiva no sangue periférico, o que faz pensar em cura radical mesmo com esquema de primaquina por tempo reduzido.

\footnotetext{
1. Núcleo de Medicina Tropical da Universidade Federal do Pará, Belém, PA. 2. Instituto Evandro Chagas da Fundação Nacional de Saúde Endereço para correspondência: Dr. José Maria de Souza. Programa de Malária/IEC. Av. Almirante Barroso, 492, 66090-000 Belém, PA.

Tel: 5591 211-4432; Fax: 5591 211-4463/226-1284

E-mail :jmsouza@iec.pa.gov.br/ayece@iec.pa.gov.br

Recebido para publicação em 25/04/2000.
} 
Estas observações preliminares, despertaram o interesse na busca de um esquema terapêutico que mesmo em tempo reduzido, proporcionasse ao paciente maior adesão ao tratamento e, ao mesmo tempo, eficácia e tolerância, minimizando efeitos colaterais e reduzindo custos ${ }^{1420}$.
Os autores se propõem avaliar a eficácia, tolerância e toxicidade de três diferentes esquemas terapêuticos para o tratamento da malária por $P$. vivax e registrar os possíveis efeitos colaterais ou tóxicos produzidos pela primaquina em dose dobrada.

\section{MATERIAL E MÉTODOS}

O estudo foi realizado no Ambulatório do Programa de Malária do Instituto Evandro Chagas (APM/IEC) em Belém/PA, considerado uma unidade de referência no Estado do Pará para tratamento da malária. Caracterizou-se por ser um estudo aberto, prospectivo e randomizado.

Os critérios de inclusão obedecidos foram: diagnóstico parasitológico confirmado para malária por $P$. vivax; idade superior a 12 anos e permanência em Belém até o final do controle de cura (180 dias). Foram excluídos gestantes e nutrizes; pacientes que utilizaram antimaláricos há pelo menos 2 semanas antes do início do tratamento atual; portadores de malária mista.

Após inclusão no estudo, os pacientes eram alocados em três grupos (I, II, III), cada um deles composto de 40 participantes assim distribuídos: grupo I: cloroquina, comprimidos contendo $150 \mathrm{mg}$ de cloroquina base, na dose de $25 \mathrm{mg} / \mathrm{kg}$ como dose total, dividida em 3 dias, sendo, $10 \mathrm{mg} / \mathrm{kg}$ de peso por dia no primeiro dia, $7,5 \mathrm{mg} / \mathrm{kg}$, no segundo e terceiro dias, associada ao fosfato de primaquina, em comprimidos contendo $15 \mathrm{mg}$ de substância base, na dose de $0,25 \mathrm{mg} / \mathrm{kg} / \mathrm{dia}$, administrada por 14 dias seguidos; grupo Il: cloroquina, na dose de $10 \mathrm{mg} / \mathrm{kg}$ em dose única, associada a primaquina, na dose $0,5 \mathrm{mg} / \mathrm{kg} /$ dia por sete dias seguidos; grupo III: cloroquina, na dose de 10mg/ $\mathrm{kg}$ em dose única, associada a primaquina, na dose de $0,5 \mathrm{mg} / \mathrm{kg} / \mathrm{dia}$ por cinco dias seguidos.

A medicação foi administrada sob supervisão médica, por via oral, feita diariamente durante o retorno do paciente por ocasião da avaliação clínico-laboratorial, na primeira semana.

A cura clínica foi considerada como sendo o desaparecimento dos principais sintomas e sinais de malária (febre, frio e cefaléia), após introdução da terapêutica. A cura parasitológica foi considerada como o desaparecimento da parasitemia assexuada a partir do início da terapêutica específica, até um período de acompanhamento de no mínimo trinta dias. Cura radical foi considerada como sendo a persistência da cura parasitológica por um acompanhamento prolongado de 180 dias. Como efeitos colaterais foram considerados quaisquer sintomas ou sinais inexistentes antes da administração das drogas que tenham surgido após sua utilização.

O exame de gota espessa era realizado diariamente até a detecção de dois exames negativos consecutivos, seguindo-se um exame no final da primeira semana de tratamento (D7) e nos dias do controle de cura parasitológico, realizado através de um exame de gota espessa, mensalmente durante seis meses seguidos (180 dias).

A digitação e sistematização das informações foram realizadas em banco de dados próprio, produzido em microcomputador e programa EPI-INFO, versão 6.04.

O tempo de desaparecimento da tríade sintomática e da parasitemia assexuada foi avaliado pelo teste do qui-quadrado, efeitos colaterais foram avaliados pelo Teste Exato de Fisher. Tempo de recaída, avaliado pela análise de variância (Kruskal-Wallis $\mathrm{H}$ ). Foram considerados significantes resultados de análises cujos valores de $p$ foram menores que 0,05 .

\section{RESULTADOS}

A amostra foi constituída por 120 pacientes com malária por P. vivax, sendo 40 em cada grupo de estudo, tratados no período de julho de 1994 a junho de 1995.

Os dados pessoais e biométricos da amostra estudada encontram-se na Tabela 1.

Nenhum paciente apresentou complicações, sendo todos tratados em nível ambulatorial. Em 119 (99,2\%) pacientes pode-se determinar o tempo decorrido entre o início da sintomatologia e a realização do diagnóstico no Instituto Evandro Chagas, que foi em média de 8 dias, tendo variado de 0 a 48 dias.

Cento e dez $(91,7 \%)$ indivíduos referiram a tríade (febre, calafrios e cefaléia) no primeiro dia de tratamento. Em D1 (cerca de 24 horas a partir do início do tratamento), 25,5\% destes estavam livres destes sinais e sintomas, em D2 (cerca de quarenta e oito horas após) este percentual elevou-se para $81 \%$ e em D3 (cerca de 72 horas após) atingiu a quase a totalidade dos casos $(98,2 \%)$. Não houve diferenças estatisticamente significativas entre a proporção de indivíduos assintomáticos e sintomáticos em cada grupo, conforme dados encontrados na Tabela 2.

Em 23 pacientes, houve o registro de efeitos colaterais $(19,2 \%$ da amostra), encontrados em 11 pacientes do grupo I e em 6 dos grupos II e III. Os efeitos adversos relatados foram: diarréia $(5,8 \%)$, náuseas $(5 \%)$, prurido $(5 \%)$, vômitos $(1,7 \%)$, epigastralgia $(1,7 \%)$, tonturas $(0,8 \%)$ e zumbidos $(0,8 \%)$. Não houve necessidade de interrupção do tratamento em nenhum dos casos. 
Tabela 1 - Dados pessoais e biométricos dos 120 pacientes com malária por $\mathrm{P}$. vivax submetidos ao tratamento com três esquemas terapêuticos. Belém-Pará, 1994-1995.

\begin{tabular}{|c|c|c|c|c|}
\hline \multirow[t]{2}{*}{ Dados pessoais e biométricos } & \multicolumn{3}{|c|}{ Grupos } & \multirow[b]{2}{*}{$\mathrm{p}$} \\
\hline & I & II & III & \\
\hline Número de pacientes & 40 & 40 & 40 & \\
\hline Sexo (\% masculino) & 60 & 65 & 62,5 & 0,8988 * \\
\hline \multicolumn{5}{|l|}{ Peso corporal (kg) } \\
\hline média & 56,96 & 63,58 & 57,63 & 0,0897 ** \\
\hline amplitude & $28-100$ & $40-110$ & $30-100$ & \\
\hline \multicolumn{5}{|l|}{ Idade (anos) } \\
\hline média & 27 & 31 & 24 & $0,0255^{* *}$ \\
\hline amplitude & $12-67$ & $12-66$ & $12-51$ & \\
\hline \multicolumn{5}{|l|}{ Cor da pele (\%) } \\
\hline faioderma & 40,0 & 57,5 & 47,5 & $0,4084^{*}$ \\
\hline branca & 52,5 & 35,0 & 50,0 & \\
\hline negra & 7,5 & 7,5 & 2,5 & \\
\hline
\end{tabular}

* Qui-quadrado $\left(\mathrm{c}^{2}\right)$

** Análise de variância (Kruskal Wallis $\mathrm{H}$ )

Tabela 2 - Desaparecimento da tríade malárica (febre, calafrios e cefaléia) em 110 pacientes com malária por P. vivax, incluídos no estudo, conforme grupo de tratamento, Belém-Pará, 1994-1995.

\begin{tabular}{lcrr}
\hline Tempo de desaparecimento & \multicolumn{3}{c}{ Pacientes assintomáticos por grupos de tratamento (\%) } \\
\cline { 2 - 4 } dos sintomas* $^{*}$ & \multicolumn{1}{c}{ I } & 9 & III \\
\hline D1 & 13 & 20 & 6 \\
D2 & 17 & 6 & 24 \\
D3 & 7 & 1 & 6 \\
D4 & 0 & 36 & 37 \\
\hline Total & 37 & & 37 \\
\hline
\end{tabular}

Qui-quadrado- $p=0,5035$

*10 pacientes não apresentaram a tríade.

A parasitemia assexuada inicial variou de 40 a 17.500 parasitas por milímetro cúbico de sangue, e pode ser vista na Figura 1, para os diferentes grupos de tratamento, que foram considerados homogêneos quanto a parasitemia assexuada no primeiro dia de tratamento. (Análise de variância - Kruskal Wallis H: $\mathrm{p}=0,3024$ ).
Todos os pacientes apresentaram negativação da parasitemia assexuada no máximo com 72 horas após ser instituída a terapêutica. A maioria $(77,5 \%)$ dos pacientes apresentou desaparecimento da parasitemia assexuada ao exame de gota espessa, em cerca de 48 horas. Não ocorreram diferenças estatisticamente

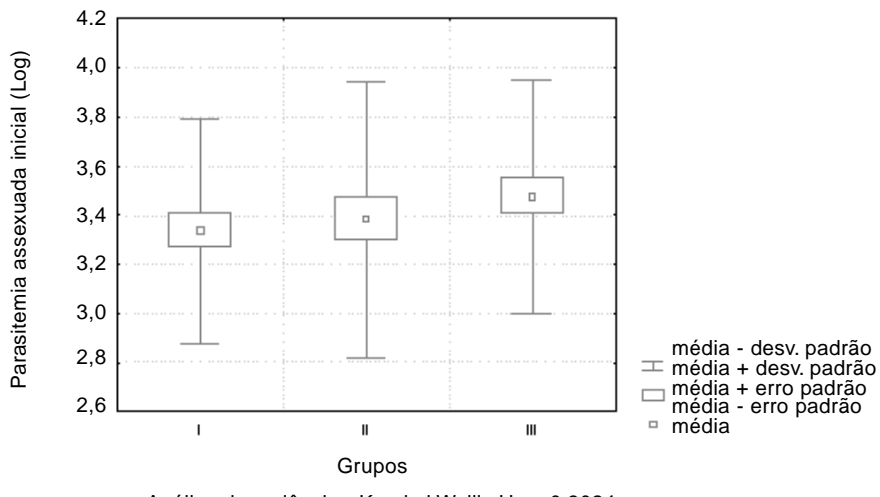

Análise de variância - Kruskal Wallis $\mathrm{H}: \mathrm{p}=0.3024$

Figura 1 - Parasitemia assexuada inicial dos 120 pacientes com malária por $\mathrm{P}$. vivax submetidos ao tratamento com três distintos esquemas terapêuticos, Belém, Pará, 1994-1995. 
significativas quanto a esta variável nos pacientes incluídos nos três diferentes grupos estudados conforme mostra a Tabela 3.

Trinta e oito (95\%) pacientes tratados com o esquema clássico curaram e dois (5\%) apresentaram recaída; nos indivíduos que foram alocados no esquema II, a cura ocorreu em 39 (97,5\%) deles e um (2,5\%) abandonou o controle de 180 dias (o paciente recebeu tratamento completo, porém seu último controle foi realizado em D7); nas pessoas que foram submetidas ao tratamento com o esquema III, trinta e duas (80\%) curaram e oito (20\%) recaíram.

Quanto aos percentuais de cura, não houve diferenças estatísticamente significantes entre o esquema clássico e o esquema II, enquanto que o esquema III mostrou-se menos eficaz que os dois anteriores (Tabela 4)

\begin{tabular}{|c|c|c|c|c|}
\hline \multirow[b]{2}{*}{ Dia de Negativação } & \multicolumn{3}{|c|}{ № de Pacientes por Esquema } & \multirow[b]{2}{*}{ Total de pacientes (\%) } \\
\hline & I & II & III & \\
\hline $\mathrm{D} 1$ & 6 & 8 & 5 & 15,8 \\
\hline D2 & 28 & 22 & 24 & 61,7 \\
\hline D3 & 6 & 10 & 11 & 22,5 \\
\hline Total & 40 & 40 & 40 & 100,0 \\
\hline
\end{tabular}

Qui-quadrado- $\mathrm{p}=0,5496$.

Tabela 4 - Respostas terapêuticas observadas nos 120 pacientes com malária por P. vivax submetidos ao tratamento com três distintos esquemas terapêuticos, Belém-Pará, 1994-1995.

\begin{tabular}{|c|c|c|c|c|c|c|}
\hline \multirow[t]{3}{*}{ Respostasterapêuticas } & \multicolumn{6}{|c|}{ Esquemas } \\
\hline & \multicolumn{2}{|c|}{1} & \multicolumn{2}{|c|}{ II } & \multicolumn{2}{|c|}{ III } \\
\hline & $\mathrm{n}^{0}$ & $\%$ & $\mathrm{n}^{\circ}$ & $\%$ & $\mathrm{n}^{0}$ & $\%$ \\
\hline Cura & 38 & 95,0 & 39 & 97,5 & 32 & 80,0 \\
\hline Recaída & 2 & 5,0 & 0 & 0,0 & 8 & 20,0 \\
\hline Abandono de controle & 0 & 0,0 & 1 & 2,5 & 0 & 0,0 \\
\hline
\end{tabular}

Teste Exato de Fisher: entre I e II : $p=0,2532$; entre I e III: $p=0,0436$; entre II e III: $p=0,0030$.

\section{DISCUSSÃO}

O tratamento da malária produzida pelo $P$ vivax, vem sendo realizado no Brasil, com cloroquina por três dias associada a primaquina por quatorze dias, e sua eficácia tem se mantido até hoje, embora possa ser imputado à sua duração, o grande índice de abandono e conseqüente falha no tratamento, resultando em recaídas da doença.

Ao iniciar a terapêutica específica, notou-se que a tríade sintomática da malária, representada por febre, calafrio e cefaléia, que ocorreu como manifestação inicial em 110 pacientes, desapareceu, 24 horas após o início do tratamento em $28(25,5 \%)$ indivíduos dos quais $15(53,56 \%)$ pertenciam aos grupos que utilizaram a cloroquina em dose única. Em D4 todos 110 pacientes estavam assintomáticos para a tríade. Dessa maneira, os autores consideram que tanto a cloroquina, usada por três dias como em dose única, foi capaz de provocar desaparecimento da sintomatologia independentemente do tempo de uso do medicamento. Em estudo comparativo entre a cloroquina, em dose única e o artesunato por via oral no tratamento da malária vivax, foi demonstrado que $50 \%$ dos casos tratados com cloroquina, tornaram-se assintomáticos para a tríade malárica em até 48 horas $^{20}$.

Os efeitos colaterais observados na amostra foram de pequena intensidade $(19,2 \%)$ e efêmeros, sem necessidade de intervenção. A maioria desses efeitos ocorreu em relação ao aparelho gastrintestinal, tendo como manifestação principal a diarréia que figurou em $5,8 \%$ dos casos. O prurido ocorreu em $6(5 \%)$, pacientes e destes, $4(66,7 \%)$ pertenciam ao grupo que utilizou a cloroquina por três dias. Vários autores já relatavam o prurido devido ao uso da cloroquina, sobretudo quando a droga é utilizada por três dias, o que está de acordo com o que foi encontrado no presente estudo ${ }^{1311}$. Nagarathnam et al, descreveram três casos de anemia aplástica associada ao uso da cloroquina, entre os quais, um deles desenvolveu anemia aplástica três semanas após um curto tratamento para malária ${ }^{10}$. Assim, ao se optar por um tratamento reduzido, o risco da ocorrência desse efeito tóxico sobre o tecido hematopoiético será ainda menor.

$\mathrm{Na}$ Tailândia, a fim de obter melhor resposta no tratamento da malária vivax Bunnag et al usaram a 
primaquina na dose de $22,5 \mathrm{mg}$ por dia por 14 dias, e não encontraram efeitos adversos ${ }^{5}$. Em 1977 em trabalho onde foi utilizada a primaquina $60 \mathrm{mg} / \mathrm{dia}$ por sete dias, no tratamento da malária vivax induzida, foram relatados efeitos gastrointestinais intestinais importantes ${ }^{6}$. Já o emprego de primaquina em doses 10 vezes superiores a convencional ou seja $2,5 \mathrm{mg} / \mathrm{kg}$ de peso corporal por dois dias nas Guianas não registraram efeito tóxico ${ }^{13}$. O exame clínico dos casos ora estudados mostrou que mesmo duplicando a dose da primaquina, não ocorreram efeitos colaterais ou sinais de toxicidade mais grave.

A parasitemia assexuada desapareceu em até no máximo 72 horas após o início do tratamento nos três esquemas, sugerindo que a cloroquina em dose única é capaz de eliminar a parasitemia assexuada do sangue periférico, dispensando seu uso por mais dois dias, em conformidade com os achados de outros autores, que registraram velocidade média de negativação da parasitemia assexuada de 51,7 horas, quando utilizaram a cloroquina em dose única $(600 \mathrm{mg})$ para tratar 60 pacientes adultos com malária vivax e também um outro estudo, realizado em 200 crianças com malária vivax, sob tratamento com dose única de cloroquina, obteve-se cura clínica em todas elas até o quarto dia de tratamento ${ }^{14} 20$.

Ao estudarmos doses de antimaláricos, obrigatoriamente é necessário o conhecimento prévio dos estudos em resistência plasmodial às drogas. Já está bem documentada a resistência do $P$. falciparum às drogas antipalúdicas ${ }^{12}$, e mais recentemente, a literatura médica tem se voltado para a resistência do $P$. vivax à cloroquina, já relatada em trabalhos realizados na Ásia (Papua Nova Guiné, Ilhas Salomão, Indonésia) ${ }^{161721 .}$

As primeiras evidências de que o P.vivax estivesse desenvolvendo resistência à cloroquina, foi relatada em uma criança de 8 meses de idade, procedente Papua Nova Guiné, portadora de malária mista ${ }^{17}$.

Foram descritos dois casos de pacientes sob profilaxia contínua, com $300 \mathrm{mg}$ de cloroquina base que não obtiveram proteção para malária vivax em Papua Nova Guiné, embora as concentrações de cloroquina no plasma estivessem bem acima das necessárias para supressão da malária vivax ${ }^{15}$. Os autores estabeleceram características morfológicas para diferenciar o $P$. vivax do $P$. ovale ${ }^{15}$, entretanto, este critério não foi adotado para diferenciar os trofozoítos do $P$. falciparum, cuja semelhança com o $P$. vivax pode gerar dúvidas com relação à autenticidade da resistência.
Em 1946, estabeleceu-se que concentrações de cloroquina de $15 \mu \mathrm{g} / \mathrm{l}$ no plasma e $30 \mu \mathrm{g} / \mathrm{l}$ no soro, são suficientes para proporcionar supressão da malária vivax. O nível de cloroquina ideal deve ser dosado no sangue total e, $90 \mu \mathrm{g} / \mathrm{l}$ são suficientes para a ação desejada sobre o parasita, sendo que esta concentração no sangue é quase sempre ultrapassada após o tratamento convencional (25mg/kg de peso) para malária vivax ${ }^{489}$.

Infelizmente, não foi possível dosar os níveis de cloroquina no sangue dos pacientes envolvidos neste ensaio, entretanto os critérios clínicos (desaparecimento da tríade sintomática) e parasitológicos (negativação da parasitemia), confirmaram a eficácia da droga, mesmo em doses reduzidas a menos da metade daquela usada no esquema padrão.

Os percentuais de recaídas para o esquema I (cloroquina $25 \mathrm{mg} / \mathrm{kg}$ de peso corporal administrada por três dias, associada à primaquina por quatorze dias), foram de $5 \%$, semelhantes aos achados no Maranhão, de 5,6\% de recaídas com o uso do esquema clássico ${ }^{18}$. Entretanto, diferem dos dados obtidos em São Paulo, onde o índice de recaídas foi de $24,5 \%$ para o esquema padrão².

No esquema II (cloroquina $10 \mathrm{mg} / \mathrm{kg}$ de peso em dose única associada à primaquina por sete dias), obteve-se a cura em $97,5 \%$ dos casos, sem registro de recaídas, concordando com outros autores no estado do Pará, que observaram em 39 crianças portadoras de malária vivax utilizando um esquema de tratamento semelhante ao esquema II, um percentual de cura de $92,3 \%{ }^{14}$. Também no estado do Pará, obteve-se índice de cura de 96,7\%, no tratamento de 30 pacientes com malária vivax usando esquema terapêutico idêntico ao esquema II20.

De acordo com os resultados encontrados, o esquema encurtado envolvendo cloroquina em dose única de $600 \mathrm{mg}$ e primaquina em dose dobrada por sete dias, mostrou ser eficaz, quando analisado isoladamente, porém outros estudos são necessários para comparar com o esquema clássico, em diferentes áreas da Amazônia.

O esquema III (cloroquina $10 \mathrm{mg} / \mathrm{kg}$ de peso em dose única associada a primaquina por cinco dias), revelou ser o menos eficaz, mostrando $20 \%$ de recaídas, diferentemente do que ocorreu no Maranhão, durante o tratamento de 1.232 casos de malária por $P$. vivax, com cloroquina nas doses habituais, associada a primaquina em doses simples, por cinco dias, nos quais as recaídas aconteceram em apenas $10,2 \%$ dos casos $^{19}$. Os mesmos autores, ao reduzirem a cloroquina para a metade da dose recomendada e mantendo a primaquina em $15 \mathrm{mg}$ durante cinco dias, detectaram $4 \%$ de recaídas.

\section{REFERÊNCIAS BIBLIOGRÁFICAS}

1. Ajayi AA, Oluokum A, Sofowora O, Akinleye A, Ajayi AT. Epidemiology of antimalarial-induced pruritus in Africans. European Journal Clinical Pharmacology 37: 539-540, 1989.

2. Boulos M, Amato Neto V, Dutra AP Di Santi SA, Shiroma M. Análise da freqüência de recaídas de malária por Plasmodium vivax em região não endêmica, São Paulo, Brasil. Revista do Instituto de Medicina Tropical de São Paulo 33: 143-146, 1991.
3. Bruce-Chwatt LJ. Chemoprophylaxis of malaria in Africa [letter]. British Medical Journal 85(6354):1578-1579, 1982.

4. Bruce-Chwatt LJ, Black RH, Canfield CJ. Ed. Pharmacology of compounds in current use. In: Chemotherapy of malaria. 2. edition Geneva, World Health Organization, p. 57-91, 1981.

5. Bunnag D, Karbwang J, Thanavibul A. Chittamas S, Ratanapongse Y, Chalermrut K, Na Bangchang K, Harinasuta T. 
High dose of primaquine in primaquine resistant vivax malaria. Transactions of the Royal Society of Tropical Medicine and Hygiene 88:218-219, 1994.

6. Clyde DF, Mccarthy VC. Radical cure of Chesson strain vivax malaria in man by 7 , not 14 , days of treatment with primaquine. The American Journal of Tropical Medicine and Hygiene 26: 562563, 1977

7. Garavelli PL, Corti E. Chloroquine resistance in Plasmodium vivax: the first case in Brazil. Transactions of the Royal Society of Tropical Medicine and Hygiene 86:128, 1992.

8. Most H, London IM, Kane CA, Lavietes PH, Shroeder EF, Hayama JM. Chloroquine treatment of acute attacks of vivax malaria. Journal of the American Medical Association 131: 963-67, 1946.

9. Murphy, GS, Barsi H, Purnomo, Andersen EM, Bangs MJ, Mount DL, Gorden JL, Lal AA, Purwokusumo AR, Harjosuwarno S. Vivax malaria resistant to treatment and prophylaxis with chloroquine. The Lancet 341:96-100, 1993.

10. Nagarathnam N, Chetiyawardana AD, Rajiyah S. Aplasia and leukaemia following chloroquine therapy. Postgraduat Medical Journal, 54:108-112, 1978.

11. Osifo, NG. Chloroquine-induced pruritus among patients with malaria. Archives of Dermatology 120: 80-82, 1984.

12. Peters, W. Chemotherapy and Drug Resistance in Malaria. 2 volumes, 2nd edition. Academic Press, London pp, 10, 579-585, 595-599, 620-626, and 10561987.

13. Phillips EJ, Keystone JS, Kain KC. Failure of combined Chloroquine and high - dose primaquine therapy for Plasmodium vivax malaria acquired in Guyana, South America. Clinical Infectious Diseases 23:1173, 1996
14. Pinto AYN, Ventura AMS, Calvosa VSP, Silva Filho MG, Santos MA, Silva RSU, Souza JM. Eficácia clínica e terapêutica de quatro esquemas no tratamento da malária vivax em crianças. Jornal de Pediatria 74:222-227, 1998.

15. Rieckmann KH, Davis DR, Hutton DC. Plasmodium vivax resistance to chloroquine? The Lancet 2:1183-1184, 1989.

16. Schuurkamp GJ, Spicer PE, Kereu RK, Buungol PK, Rieckmann $\mathrm{KH}$. Chloroquine-resistant Plasmodium vivax in Papua New Guinea. Transactions of the Royal Society of Tropical Medicine and Hygiene 86:121-122, 1992.

17. Schwartz IK, Lackritz EM, Patchen LC. Chloroquine-resistant Plasmodium vivax from Indonesia. The New England Journal of Medicine 324:927, 1991.

18. Silva AR, Carneiro EWB, Santos HJ. Resposta dos plasmódios humanos aos antimaláricos na llha de São Luiz, Estado do Maranhão, Brasil. Revista do Instituto de Medicina Tropical de São Paulo 26:139-146, 1984.

19. Silva AR, Silva CMP, Carvalho Branco MRF, Branco Filho JRC. Resultado do uso de um esquema terapêutico para Plasmodium vivax em cinco dias em três municípios da llha de São Luiz, Estado do Maranhão, Brasil. Revista da Sociedade Brasileira de Medicina Tropical 22:131-136, 1989.

20. Uchôa RS, Souza JM, Pinto AYN, Calvosa VSP, Abdon NPA, Silva DR, Melo ES, Meireles R. Maior operacionalidade no tratamento da malária vivax. In: Resumos do XXX Congresso da Sociedade Brasileira de Medicina Tropical, Salvador, p. 271, 1994,

21. Whitby M, Wood G, Venendaal Jr, Rieckmann K. Chloroquineresistant Plasmodium vivax. The Lancet 2:1395, 1989. 